\title{
Outage Probability Analysis of Full-Duplex Regenerative Relaying over Generalized Asymmetric Fading Channels
}

\author{
Mulugeta K. Fikadu ${ }^{1}$, Paschalis C. Sofotasios ${ }^{1,2}$, Mikko Valkama ${ }^{1}$, Qimei Cui ${ }^{3}$, Sami Muhaidat ${ }^{4,5}$, \\ and George K. Karagiannidis ${ }^{2,4}$
${ }^{1}$ Department of Electronics and Communications Engineering, Tampere University of Technology, 33101 Tampere, Finland. e-mail: \{mulugeta.fikadu; paschalis.sofotasios; mikko.e.valkama\} @tut.fi
${ }^{2}$ Department of Electrical and Computer Engineering, Aristotle University of Thessaloniki, 54124 Thessaloniki, Greece. e-mail: geokarag@auth.gr
${ }^{3}$ Wireless Technology Innovation Institute, Beijing University of Posts and Telecommunications, 100876 Beijing, China. e-mail: cuiqimei@bupt.edu.cn
${ }^{5}$ Department of Electronic Engineering, University of Surrey, GU2 7XH, Guildford, United Kingdom. \\ ${ }^{4}$ Department of Electrical and Computer Engineering, Khalifa University, PO Box 127788, Abu Dhabi, United Arab Emirates. \\ e-mail: muhaidat@ieee.org
}

\begin{abstract}
This work is devoted to the outage probability analysis of full-duplex (FD) regenerative relay systems over multipath fading channels. Unlike the majority of analyses that assume basic symmetric fading conditions, the present work considers asymmetric generalized fading conditions, which are more realistic in practical communications scenarios. To this end, we assume that the source-relay path is subject to $\kappa-\mu$ multipath fading conditions, that can also account for lineof-sight communications, whereas the source-to-destination and relay-to-destination paths are subject to $\eta-\mu$ fading conditions that typically hold for non-line-of-sight communications. Novel analytic expressions are derived for the outage probability (OP) of the considered FD as well as for the corresponding halfduplex (HD) relay case for comparisons. These expressions are given in closed-form and have a tractable algebraic representation which renders them convenient to handle both analytically and numerically. Based on this, they are subsequently employed in analyzing the corresponding performance for different communication scenarios. It is shown that the OP of the FD relay system is highly dependent upon the severity of fading and that its performance outperforms significantly the corresponding HD performance as the spectral efficiency increases.
\end{abstract}

\section{INTRODUCTION}

Cooperative communications have attracted significant interest over the past decade due to their potential to expand cellular network coverage, increasing channel capacity and reduce power consumption of wireless devices [1]. Traditionally, relay systems are assumed to operate in half-duplex (HD) mode where the relay receives and transmits on orthogonal channels [2]-[8] and the references therein. However, although HD architectures are considered relatively simpler in terms of system design and implementation requirements, they also suffer by inherent spectral efficiency loss. On the contrary, full-duplex relaying has been proposed as an effective alternative architecture that is capable of overcoming this critical inefficiency by allowing relay(s) to receive and transmit simultaneously at the same frequency band, see e.g. [9] and the references therein.

However, full-duplex communications are practically susceptible to the inherent relay self-interference (RSI) due to the concurrent transmission and reception. Yet, recent studies have shown that FD relaying strategies are feasible even in the presence of high self-interference levels [10]. Motivated by this, the authors in [11] derived closed-form expressions for the outage probability (OP) over Rayleigh fading channels by considering the relay self-interference. Likewise, the authors in [12] analyzed the performance of full-duplex block Markov relaying with self-interference at the relay over independent but non-identically distributed Nakagami $-m$ fading channels. A comprehensive OP analysis in a three-terminal full-duplex relay system for a selective decode-and-forward (DF) protocol with RSI was studied in [13] whereas the authors in [14] proposed hybrid techniques that switch opportunistically between FD and HD relaying modes in both DF and amplifyand-forward (AF) systems.

Nevertheless, all reported investigations on FD relaying assume information transmission over symmetric multipath fading channels, i.e., that the source-relay and relay-destination links undergo the same fading conditions. However, this assumption is rather simplistic since in realistic communication scenarios, different wireless paths are subject to non identical fading channels while they experience generalized fading conditions [15]-[18] and the references therein. For example, communication in one path might be line of sight 


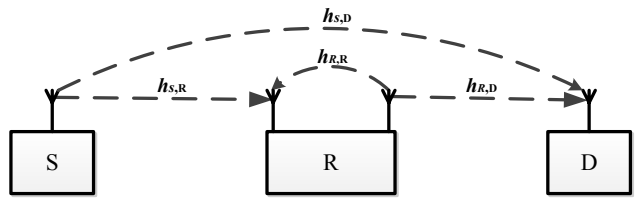

Fig. 1. Two-hop full-duplex relay system.

(LOS) with the presence of a dominant component while another path might experience severe non-line of sight NLOS conditions. Based on this, the authors in [19] investigated the performance of repetition-based two hop HD DF system over asymmetric Rayleigh and Rician fading channels whereas the authors in [20] analyzed the performance of dual-hop HD AF relay networks over asymmetric Rayleigh and Rician fading conditions. In the same context, the authors in [21] investigated the end-to-end performance of a dual-hop asymmetric HD AF system where the source-to-relay path is subject to $\eta-\mu$ or $\kappa-\mu$ fading while the relay-to-destination channel is subject to $\kappa-\mu$ or $\eta-\mu$ fading conditions.

However, in spite of the usefulness of FD relaying, the majority of analyses over asymmetric fading conditions are considered only on half-duplex relaying systems. Motivated by this, the present work analyzes the OP in FD relay systems over generalized asymmetric fading channels. To this end, novel analytic expressions are derived for the corresponding OP for Rayleigh distributed RSI. Also, $\kappa-\mu$ fading conditions are assumed for the source-to-relay path while $\eta-\mu$ fading conditions are assumed for the source-to-destination and relayto-destination paths. The offered analytic results are expressed in closed-form and their validity is justified through comparisons with respective results from computer simulations. The derived expressions are subsequently employed in analyzing the OP for different scenarios and as expected, it is shown that the performance of the considered FD relaying is highly dependent upon the value of the fading parameters. Furthermore, extensive comparisons with the corresponding HD scenario indicates that the FD architecture outperforms significantly the HD counterpart particularly for high spectral-efficiency values.

\section{System ANd Channel Model}

\section{A. System model of Full-Duplex Relay}

We consider a two-hop network that consists of a single antenna source node $\mathrm{S}$, a relay node $\mathrm{R}$ operating in FD mode and a single antenna destination node $\mathrm{D}$ as illustrated in Fig.1. The considered cooperative strategy is based on the DF protocol where the source transmits the information signal to the relay node and the relay node receives this signal and re-transmits towards the destination. In the FD mode the re-transmission occurs in the same frequency band, which ultimately induces self interference. The received signals at the relay and destination nodes can be expressed as

$$
y_{R}=\sqrt{P_{S}} h_{S, R} x_{S}+\sqrt{P_{R}} h_{R, R} x_{R}+n_{R}
$$

and

$$
y_{D}=\sqrt{P_{R}} h_{R, D} x_{R}+\sqrt{P_{S}} h_{S, D} x_{S}+n_{D}
$$

respectively, where $P_{S}$ and $P_{R}$ are the transmission powers at the source and relay nodes, respectively, $x_{S}$ and $x_{R}$ denote the transmitted signals from the source and relay nodes with normalized unit energy, whereas $h_{S, R}, h_{R, D}, h_{S, D}$ and $h_{R, R}$ are the fading coefficients of the $\mathrm{S} \rightarrow \mathrm{R}, \mathrm{R} \rightarrow \mathrm{D}, \mathrm{S} \rightarrow \mathrm{D}$ and $\mathrm{R} \rightarrow \mathrm{R}$ links, respectively. Also, $n_{R}$ and $n_{D}$ denote the additive white Gaussian noise (AWGN) with zero mean and variance $N_{0}$ at the relay and destination nodes, respectively while each path experiences narrow band multipath fading.

\section{B. Asymmetric Channel Model}

As already mentioned, fading conditions in asymmetric channels differ between at least two paths of the system. Based on this, in the present analysis we assume that the $S \rightarrow R$ path experiences $\kappa-\mu$ fading conditions whereas the $R \rightarrow D$ and $S \rightarrow D$ paths are subject to $\eta-\mu$ fading conditions. It is noted that these fading models have been shown to be remarkably accurate for modeling multipath fading in LOS and NLOS communications, respectively [22]. Furthermore, the relay self-interference channel is assumed to be Rayleigh distributed, which corresponds to severe fading conditions.

1) The $\eta-\mu$ fading model: The probability density function of the $\eta-\mu$ fading distribution is expressed as [22]

$$
f_{\gamma}(\gamma)=\frac{2 \sqrt{\pi} \mu^{\mu+\frac{1}{2}} h^{\mu} \gamma^{\mu-\frac{1}{2}} I_{\mu-\frac{1}{2}}\left(\frac{2 \mu H \gamma}{\bar{\gamma}}\right)}{\Gamma(\mu) H^{\mu-\frac{1}{2}} \bar{\gamma}^{\mu+\frac{1}{2}} \exp \left(\frac{2 \mu \gamma h}{\bar{\gamma}}\right)}
$$

where $\gamma=|h|^{2} P / N_{0}$ denotes the instantaneous SNR, $\bar{\gamma}=$ $\mathrm{E}[\gamma]$ is the average SNR per symbol, whereas $\Gamma(\cdot)$ and $I_{\nu}(\cdot)$ denote the gamma function and the modified Bessel function of the first kind, respectively [23]. The parameters $h$ and $H$ correspond to the two distinct formats of the $\eta-\mu$ distribution. In Format- $1, h=\left(2+\eta^{-1}+\eta\right) / 4$ and $H=\left(\eta^{-1}-\eta\right) / 4$ where $0<\eta<\infty$ is the scattered-waves power ratio between the inphase and quadrature components of each multipath cluster. In Format- $2, h=1 /\left(1-\eta^{2}\right)$ and $H=\eta /\left(1-\eta^{2}\right)$ where $-1<\eta<1$ represents the correlation coefficient between the in-phase and quadrature components of the scattered waves in each multipath cluster. Finally, in both formats, $\mu=\mathrm{E}^{2}(\gamma)\left(1+(H / h)^{2}\right) / 2 \mathrm{~V}(\gamma)$ is related to the multipath clustering, with $\mathrm{E}(\cdot)$ and $\mathrm{V}(\cdot)$ denoting statistical expectation and variance operations, respectively. It is recalled that the $\eta-\mu$ fading model includes as special cases the Nakagami $-m$, Nakagami $-q$ (Hoyt) and Rayleigh distributions [22].

2) The $\kappa-\mu$ fading model: The instantaneous SNR PDF of $\kappa-\mu$ fading model is given by [22]

$$
f_{\gamma}(\gamma)=\frac{\mu(\kappa+1)^{\frac{(\mu+1)}{2}} \gamma^{\frac{(\mu-1)}{2}} I_{\mu-1}\left(2 \mu \sqrt{\frac{\kappa(\kappa+1) \gamma}{\bar{\gamma}}}\right)}{\kappa^{\frac{(\mu-1)}{2}} \bar{\gamma} \frac{(\mu+1)}{2} \exp \left(\mu \kappa+\frac{\mu(\kappa+1) \gamma}{\bar{\gamma}}\right)}
$$

where $\kappa>0$ denotes the ratio between the total power of the dominant components to that of the scattered waves whereas $\mu=\mathrm{E}^{2}(\gamma)(1+2 \kappa) / \mathrm{V}(\gamma)(1+\kappa)^{2}$ is related to the multipath clustering. The $\kappa-\mu$ fading model includes the Nakagami $-m$, the Nakagami- $n$ (Rice) and Rayleigh fading models as special cases while its corresponding CDF is given by [22, eq. (3)]. 


\section{Outage Probability Analysis}

In order to derive a closed-form expression for the OP over asymmetric $\kappa-\mu$ and $\eta-\mu$ fading channels in both DF and $\mathrm{HD}$ relaying, it is necessary to employ an alternative closedform expression for the CDF of the $\eta-\mu$ distribution.

\section{A. A Simple Expression for the CDF of $\eta-\mu$ Distribution}

It is recalled that the cumulative distribution function (CDF) of a continuous distribution is defined as $F_{\gamma}(z) \triangleq \int_{0}^{z} f_{\gamma}(x) \mathrm{dx}$. To this effect, by expressing the $I_{n}(\cdot)$ in (3) according to [23, eq. (8.467], as well as using [23, eq. (8.350.1)] and carrying out long but basic algebraic manipulations, it follows that

$$
\begin{aligned}
F(z) & =\sum_{l=0}^{\mu-1} \frac{(-1)^{l} \Gamma(\mu+l)(h-H)^{l-\mu} \gamma\left(\mu-l, \frac{2(h-H) \mu z}{\bar{\gamma}}\right)}{l ! 2^{\mu+l} h^{-\mu} \Gamma(\mu) H^{\mu+l} \Gamma(\mu-l)} \\
& +\sum_{l=0}^{\mu-1} \frac{(-1)^{\mu} \Gamma(\mu+l)(h+H)^{l-\mu} \gamma\left(\mu-l, \frac{2(h+H) \mu z}{\bar{\gamma}}\right)}{l ! 2^{\mu+l} h^{-\mu} \Gamma(\mu) H^{\mu+l} \Gamma(\mu-l)}
\end{aligned}
$$

where $\gamma(a, b)$ denotes the lower incomplete gamma function. To this effect and by also using [23, eq. (8.352. 6)], the CDF of $\eta-\mu$ distribution can be equivalently expressed as follows:

$$
\begin{aligned}
F(z)= & \sum_{l=0}^{\mu-1} \frac{(-1)^{l}(h-H)^{l-\mu}+(-1)^{\mu}(h+H)^{l-\mu}}{l ! h^{-\mu} 2^{\mu+l} \Gamma(\mu) H^{\mu+l}[\Gamma(\mu+l)]^{-1}} \\
- & \sum_{l=0}^{\mu-1} \sum_{i=0}^{\mu-l-1} \frac{h^{\mu} \Gamma(\mu+l) \mu^{i} z^{i}}{l ! i ! 2^{\mu+l-i} \bar{\gamma}^{i} \Gamma(\mu) H^{\mu+l}} \\
& \times\left\{\frac{(-1)^{\mu}(h-H)^{l+i-\mu}}{\exp \left(\frac{2(h-H) \mu z}{\bar{\gamma}}\right)}-\frac{(-1)^{l}(h+H)^{l+i-\mu}}{\exp \left(\frac{2(h+H) \mu z}{\bar{\gamma}}\right)}\right\} .
\end{aligned}
$$

which is valid for integer values of $\mu$.

\section{B. $O P$ in FD Relaying}

For the DF-FD relay system, the instantaneous signal-tointerference-and-noise-ratios (SINRs) at the relay and destination nodes are expressed as follows [11], [25]

$$
\Gamma_{R}=\frac{\left|h_{S, R}\right|^{2} P_{S}}{\left|h_{R, R}\right|^{2} P_{R}+N_{0}}
$$

and

$$
\Gamma_{D}=\frac{\left|h_{R, D}\right|^{2} P_{R}}{\left|h_{S, D}\right|^{2} P_{S}+N_{0}}
$$

respectively. Here, relay is subject to self-interference while the destination is subject to interference from direct S-D signal propagation. An outage of the end-to-end communication link occurs if the $S \rightarrow R$ link can not support the required SINR or if the $R \rightarrow D$ link fails to support the required SINR. Accordingly, the overall OP of the two-hop FD relaying system can be expressed as [11]

$$
P_{\text {out }}=\operatorname{Pr}\left(\Gamma_{R}<\Gamma_{T}\right)+\left(1-\operatorname{Pr}\left(\Gamma_{R}<\Gamma_{T}\right)\right) \operatorname{Pr}\left(\Gamma_{D}<\Gamma_{T}\right)
$$

where $\Gamma_{T}=2^{R}-1$ is the required SINR with spectral efficiency $R$ in bits/sec/Hz.

For the case of asymmetric fading conditions (7) and (8) can be firstly represented in terms of the respective SNRs as $\Gamma_{R}=$ $\gamma_{S, R} /\left(\gamma_{R, R}+1\right)$ and $\Gamma_{D}=\gamma_{R, D} /\left(\gamma_{S, D}+1\right)$ respectively, where $\gamma_{R, R}$ is Rayleigh distributed with average SNR $\bar{\gamma}_{R, R}$. In order to evaluate the $\mathrm{OP}$ of the overall system, we firstly need to determine the CDFs of $\Gamma_{R}$ and $\Gamma_{D}$. To this end, by expressing $\Gamma_{R}=\gamma_{S, R} /\left(\gamma_{R, R}+1\right)$ in terms of the common RVs as $Z=X /(Y+1)$ and based on the foundations of probability theory in [26], the CDF of $Z$ can be expressed as

$$
F_{Z}(z)=\int_{0}^{\infty} F_{X}(z(y+1)) f_{Y}(y) \mathrm{d} y .
$$

To this effect, for the case of the $S \rightarrow R$ path, we substitute the CDF and PDF of $X$ and $Y$ which are the CDF of $\kappa-\mu$ distribution in [23, eq. (3)] and the PDF of Rayleigh distribution yielding

$$
F_{Z}(z)=1-\int_{0}^{\infty} \frac{Q_{\mu}\left(\sqrt{2 \kappa \mu_{S, R}}, \sqrt{\frac{2 \mu_{S, R}(1+\kappa) z(y+1)}{\bar{\gamma}_{S, R}}}\right)}{\bar{\gamma}_{R, R} \exp \left(\frac{y}{\bar{\gamma}_{R, R}}\right)} \mathrm{d} y .
$$

Importantly, a closed-form expression for (11) can be derived by setting $u=\sqrt{y+1}$ and applying [27, eq. (13)] yielding

$$
\begin{aligned}
F_{Z}(z) & =1-Q_{\mu_{S, R}}(\sqrt{b}, \sqrt{a}) \\
& +\left(\frac{a \bar{\gamma}_{S, R}}{a \bar{\gamma}_{S, R}+2}\right)^{\mu_{S, R}} \frac{Q_{\mu_{S, R}}\left(\sqrt{\frac{a b}{a+\frac{2}{\bar{\gamma}_{R, R}}}}, \sqrt{\frac{2}{\bar{\gamma}_{R, R}}+a}\right)}{e^{-\frac{1}{\bar{\gamma}_{R, R}}+\frac{b}{2+a \bar{\gamma} R, R}}}
\end{aligned}
$$

where $a=2 \mu_{S, R}(1+\kappa) z / \bar{\gamma}_{S, R}$ and $b=2 \kappa \mu_{S, R}$.

In the same context, the CDF of $\Gamma_{D}$ can be also determined with the aid of (10). To this end, by substituting the CDF and PDF of $\eta-\mu$ distribution in (6) and (3), respectively, one obtains (13), at the top of the next page. Notably, the involved integrals in (13) can be expressed in closed-form with the aid of [23, eq. (8.406.3)] and [23, eq. (6.621.1)]. Therefore, by performing the necessary variables transformation and carrying out long but basic algebraic manipulations, the CDF of $\Gamma_{D}$ can be expressed by (14), at the top of the next page.

\section{HDR Mode}

As already mentioned, conventional half-duplex regenerative relaying assumes that a signal is transmitted in two orthogonal time slots since the relay node can not transmit and receive simultaneously in the same frequency band. As a result, the self interference issue in the relay is eliminated i.e. $h_{R, R}=0$ and the corresponding OP without the direct link can be expressed as follows:

$P_{\text {out }}=\operatorname{Pr}\left(\gamma_{S, R}<\gamma_{T}\right)+\left(1-\operatorname{Pr}\left(\gamma_{S, R}<\gamma_{T}\right)\right) \operatorname{Pr}\left(\gamma_{R, D}<\gamma_{T}\right)$

where $\gamma_{T}=2^{2 R}-1$ is the required threshold value for successful transmission. Furthermore, for an asymmetric channel where fading effects in the $S \rightarrow R$ link are $\kappa-\mu$ distributed 


$$
\begin{aligned}
& F(z)=\int_{0}^{\infty}\left\{\sum_{l=0}^{\mu_{R, D}-1} \frac{h_{R, D}^{\mu_{R, D}} \Gamma\left(\mu_{R, D}+l\right)\left((-1)^{l}\left(h_{R, D}-H_{R, D}\right)^{l-\mu_{R, D}}+(-1)^{\mu_{R, D}}\left(h_{R, D}+H_{R, D}\right)^{l-\mu_{R, D}}\right)}{l ! 2^{\mu_{R, D}+l} \Gamma\left(\mu_{R, D}\right) H_{R, D}^{\mu_{R, D}+l}}\right. \\
&- \sum_{l=0}^{\mu_{R, D}-1} \sum_{i=0}^{\mu_{R, D}-l-1} \frac{(-1)^{l} h_{R, D}^{\mu_{R, D}} \Gamma\left(\mu_{R, D}+l\right) \mu_{R, D}^{i}\left(h_{R, D}-H_{R, D}\right)^{l+i-\mu_{R, D}}(z(x+1))^{i}}{l ! i ! 2^{\mu_{R, D}+l-i} \bar{\gamma}_{R, D}^{i} \Gamma\left(\mu_{R, D}\right) H_{R, D}^{\mu_{R, D}+l} \exp \left(\frac{2\left(h_{R, D}-H_{R, D}\right) \mu_{R, D} z(x+1)}{\bar{\gamma}_{R, D}}\right)} \\
&\left.-\sum_{l=0}^{\mu_{R, D}-1} \sum_{i=0}^{\mu_{R, D}-l-1} \frac{(-1)^{\mu_{R, D}} h_{R, D}^{\mu_{R, D}} \Gamma\left(\mu_{R, D}+l\right) \mu_{R, D}^{i}\left(h_{R, D}+H_{R, D}\right)^{l+i-\mu_{R, D}(z(x+1))^{i}}}{l ! i ! 2^{\mu_{R, D}+l-i} \bar{\gamma}_{R, D}^{i} \Gamma\left(\mu_{R, D}\right) H_{R, D}^{\mu_{R, D}+l} \exp \left(\frac{2\left(h_{R, D}+H_{R, D}\right) \mu_{R, D} z(x+1)}{\bar{\gamma}_{R, D}}\right)}\right\} \\
& \times \frac{2 \sqrt{\pi} \mu_{S, D}^{\mu_{S, D}+\frac{1}{2}} h_{S, D}^{\mu_{S, D}} x^{\mu_{S, D}-\frac{1}{2}}}{\Gamma\left(\mu_{S, D}\right) H_{S, D}^{\mu_{S, D}-\frac{1}{2}} \bar{\gamma}_{S, D}^{\mu_{S, D}+\frac{1}{2}}} \exp \left(-\frac{2 \mu_{S, D} x h_{S, D}}{\bar{\gamma}_{S, D}}\right) I_{\mu_{S, D}-\frac{1}{2}}\left(\frac{2 \mu_{S, D} H_{S, D} x}{\bar{\gamma}_{S, D}}\right) \mathrm{d} x .
\end{aligned}
$$

$$
\begin{aligned}
& F_{Z}(z)=\sum_{l=0}^{\mu_{R, D}-1} \frac{\Gamma\left(\mu_{R, D}+l\right)\left((-1)^{l}\left(h_{R, D}-H_{R, D}\right)^{l-\mu_{R, D}}+(-1)^{\mu_{R, D}}\left(h_{R, D}+H_{R, D}\right)^{l-\mu_{R, D}}\right)}{l ! h_{R, D}^{-\mu_{R, D}} h_{S, D}^{-\mu_{S, D}} 2^{\mu_{R, D}+l} \Gamma\left(\mu_{R, D}\right) H_{R, D}^{\mu_{R, D}+l}\left(h_{S, D}^{2}-H_{S, D}^{2}\right)^{\mu_{S, D}}}-\sum_{l=0}^{\mu_{R, D}-1} \sum_{i=0}^{\mu_{R, D}-l-1} \sum_{j=0}^{i}\left(\begin{array}{l}
i \\
j
\end{array}\right)
\end{aligned}
$$

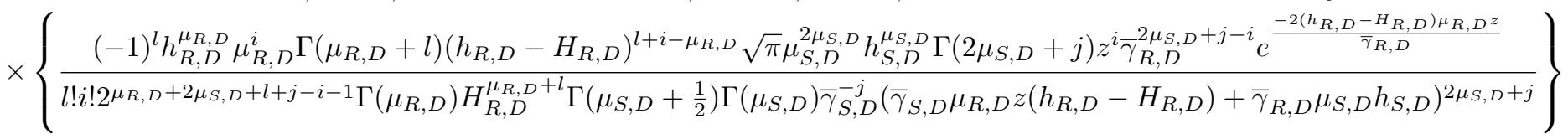

$$
\begin{aligned}
& \times_{2} F_{1}\left(\mu_{S, D}+\frac{j}{2}, \mu_{S, D}+\frac{1+j}{2}, \mu_{S, D}+\frac{1}{2}, \frac{\bar{\gamma}_{R, D}^{2} \mu_{S, D}^{2} H_{S, D}^{2}}{\left(\bar{\gamma}_{S, D} \mu_{R, D} z\left(h_{R, D}-H_{R, D}\right)+\mu_{S, D} \bar{\gamma}_{R, D} h_{S, D}\right)^{2}}\right)-\sum_{l=0}^{\mu_{R, D}-1} \sum_{i=0}^{\mu_{R, D}-l-1} \sum_{j=0}^{i}\left(\begin{array}{l}
i \\
j
\end{array}\right) \\
& \times\left\{\frac{(-1)^{\mu_{R, D}} h_{R, D}^{\mu_{R, D}} \mu_{R, D}^{i} \Gamma\left(\mu_{R, D}+l\right)\left(h_{R, D}+H_{R, D}\right)^{l+i-\mu_{R, D}} \sqrt{\pi} \mu_{S, D}^{2 \mu_{S, D}} h_{S, D}^{\mu_{S, D}} \Gamma\left(2 \mu_{S, D}+j\right) z^{i} \bar{\gamma}_{R, D}^{2 \mu_{S, D}+j-i} e^{\frac{-2\left(h_{R, D}+H_{R, D}\right) \mu_{R, D} z}{\bar{\gamma}_{R, D}}}}{l ! i ! 2^{\mu_{R, D}+2 \mu_{S, D}+l+j-i-1} \Gamma\left(\mu_{R, D}\right) H_{R, D}^{\mu_{R, D}+l} \Gamma\left(\mu_{S, D}+\frac{1}{2}\right) \Gamma\left(\mu_{S, D}\right) \bar{\gamma}_{S, D}^{-j}\left(\bar{\gamma}_{S, D} \mu_{R, D} z\left(h_{R, D}+H_{R, D}\right)+\bar{\gamma}_{R, D} \mu_{S, D} h_{S, D}\right)^{2 \mu_{S, D}+j}}\right\} \\
& \times_{2} F_{1}\left(\mu_{S, D}+\frac{j}{2}, \mu_{S, D}+\frac{1+j}{2}, \mu_{S, D}+\frac{1}{2}, \frac{\bar{\gamma}_{R, D}^{2} \mu_{S, D}^{2} H_{S, D}^{2}}{\left(\bar{\gamma}_{S, D} \mu_{R, D} z\left(h_{R, D}+H_{r d}\right)+\mu_{S, D} \bar{\gamma}_{R, D} h_{S, D}\right)^{2}}\right) .
\end{aligned}
$$

and $R \rightarrow D$ link experiences $\eta-\mu$ fading conditions, the $\operatorname{Pr}\left(\gamma_{S, R}<\gamma_{T}\right)$ and $\operatorname{Pr}\left(\gamma_{R, D}<\gamma_{T}\right)$ can be readily deduced with the aid of [22, eq. (3)] and (6), respectively.

\section{NUMERICAL RESUlTS AND Discussions}

This section employs the offered analytic results in analyzing the OP of the considered FD model over asymmetric generalized fading conditions. This is performed for different fading and interference scenarios assuming that the transmit power of the system is allocated equally to the source and relay nodes i.e. $\bar{\gamma}=\bar{\gamma}_{S, R}=\bar{\gamma}_{R, D}$.

Fig. 2 depicts the OP behavior of the FD system where the $S \rightarrow R$ link is subject to $\kappa-\mu$ fading model with fading parameters $\mu_{S, R}=1$ and $\kappa_{S, R}=\{2.5,4.5,8.5\} \mathrm{dB}$ while the $R \rightarrow D$ and $S \rightarrow D$ links are subject to the $\eta-\mu$ fading with $\mu_{R, D}=\mu_{S, D}=1$ and $\eta_{R, D}=\eta_{S, D}=1$. The mean values of the relay and the direct interference are set to $\bar{\gamma}_{R, R}=-5 \mathrm{~dB}$ and $\bar{\gamma}_{S, D}=-10 \mathrm{~dB}$ respectively for two SINR thresholds of $\Gamma_{T}=0 \mathrm{~dB}$ and $\Gamma_{T}=5 \mathrm{~dB}$. As expected, the $\mathrm{OP}$ of the asymmetric FD relaying improves as
$\kappa_{S, R}$ increases while it also observed that the OP degrades as the target $\Gamma_{T}$ increases. This is because, the system remains in complete outage until the threshold or target SINR is achieved for successful transmission.

Likewise, Fig. 3 illustrates the outage performance of the FD system as a function of the average SNR $(\bar{\gamma})$ with target SINRs of $\Gamma_{T}=0 \mathrm{~dB}$ and $5 \mathrm{~dB}$ for $\kappa_{S, R}=4.5 \mathrm{~dB}$ and $\mu_{S, D}=$ $1, \eta_{S, D}=\eta_{R, D}=1, \bar{\gamma}_{R, R}=-5 \mathrm{~dB}, \bar{\gamma}_{S, D}=-10 \mathrm{~dB}$ with different values of $\mu_{S, R}$ and $\mu_{R, D}$. It is shown that at relatively high average SNR values OP improves significantly when the values of the fading parameters $\mu_{S, R}$ and $\mu_{R, D}$ increase i.e. fading severity decreases and/or when the required threshold $\Gamma_{T}$ decreases. Thus, when the fading parameters of $S \rightarrow R$ and $R \rightarrow D$ increase, the effects of the RSI and the direct $S \rightarrow$ $D$ interference reduce and the corresponding errors tend to be negligible, which increases the performance of the system.

Fig. 4 demonstrates the OP performance as a function of the average SNR $(\bar{\gamma})$ for relatively moderate values of the fading parameter from the source to relay node and from the relay to the destination. Specifically, we set $\mu_{S, R}=\mu_{R, D}=$ 


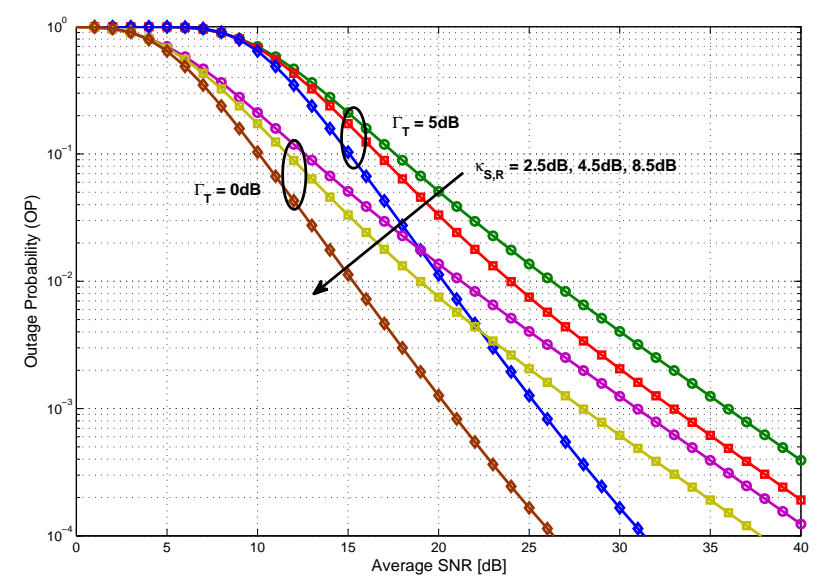

Fig. 2. Outage probability of full-duplex relaying vs. average SNR $(\bar{\gamma})$ over asymmetric $\kappa-\mu$ and $\eta-\mu$ and fading channels for $\mu_{S, R}=\mu_{R, D}=$ $\mu_{S, D}=1, \eta_{R, D}=\eta_{S, D}=1, \bar{\gamma}_{R, R}=-5 \mathrm{~dB}, \bar{\gamma}_{S, D}=-10 \mathrm{~dB}$ and different values of $\kappa_{S, R}$ and $\Gamma_{T}$.

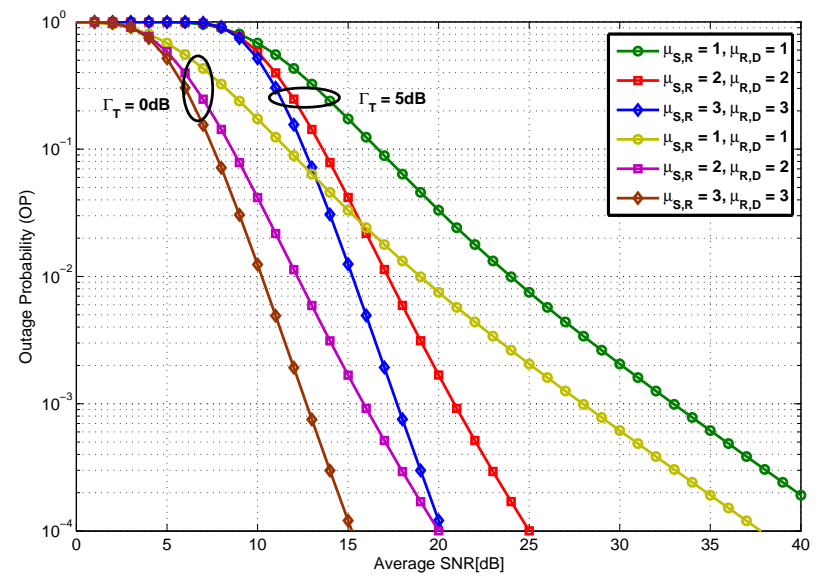

Fig. 3. Outage probability of full-duplex relaying vs. average SNR $(\bar{\gamma})$ over asymmetric $\kappa-\mu$ and $\eta-\mu$ fading channels for $\kappa_{S, R}=4.5 \mathrm{~dB}, \mu_{S, D}=$ $1, \eta_{S, D}=\eta_{R, D}=1, \bar{\gamma}_{R, R}=-5 \mathrm{~dB}, \bar{\gamma}_{S, D}=-10 \mathrm{~dB}$ and different $\mu_{S, R}=\mu_{R, D}$ and $\Gamma_{T}$ values.

$2, \mu_{S, D}=1$, and $\eta_{S, D}=\eta_{R, D}=1$ for $\bar{\gamma}_{S, D}=-10 \mathrm{~dB}$ and $\bar{\gamma}_{R, R}=\{5,0,-5\} \mathrm{dB}$ with target SINR of $\Gamma_{T}=0 \mathrm{~dB}$ and $\Gamma_{T}=5 \mathrm{~dB}$, respectively. It is shown that the performance of the FD system is significantly affected by relay selfinterference. This is because, the relay self-interference is acting as a noise to the received signal from source to relay node and thus it reduces the effective SINR and consequently the outage performance of the system. It is also noticed that, the performance degradation is not uniform as the RSI reduces from $5 \mathrm{~dB}$ to $0 \mathrm{~dB}$ and then to $-5 \mathrm{~dB}$. Hence, in order to achieve effective full duplex radio communications, the involved RSI must be canceled even in non-severe fading conditions.

Finally, Fig. 5 shows the outage performance versus $R$ for

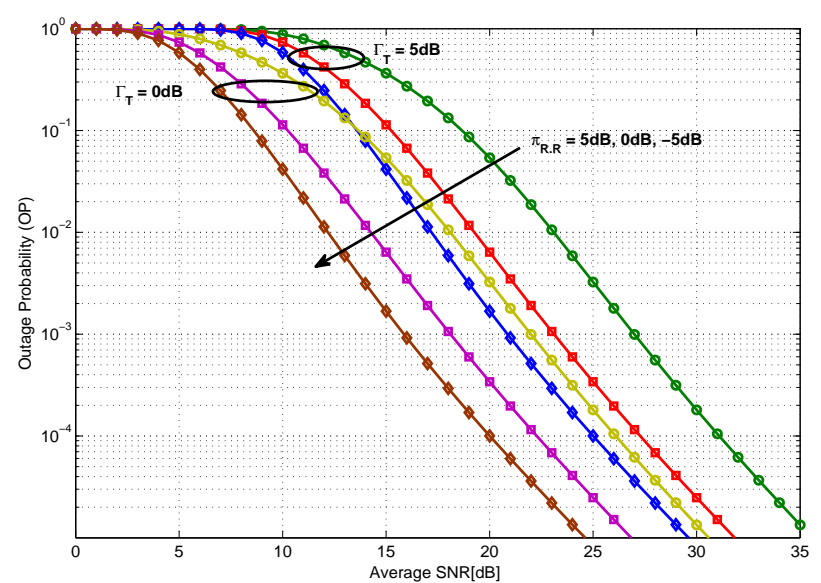

Fig. 4. Outage probability of full-duplex relaying vs. average SNR $(\bar{\gamma})$ over asymmetric $\kappa-\mu$ and $\eta-\mu$ fading channels for $\mu_{S, R}=\mu_{R, D}=2, \kappa_{S, R}=$ $4.5 \mathrm{~dB}, \mu_{S, D}=1, \eta_{S, D}=\eta_{S, R}=1, \bar{\gamma}_{S, D}=-10 \mathrm{~dB}$ and different values of $\bar{\gamma}_{R, R}=\pi_{R, R}$ and $\Gamma_{T}$.

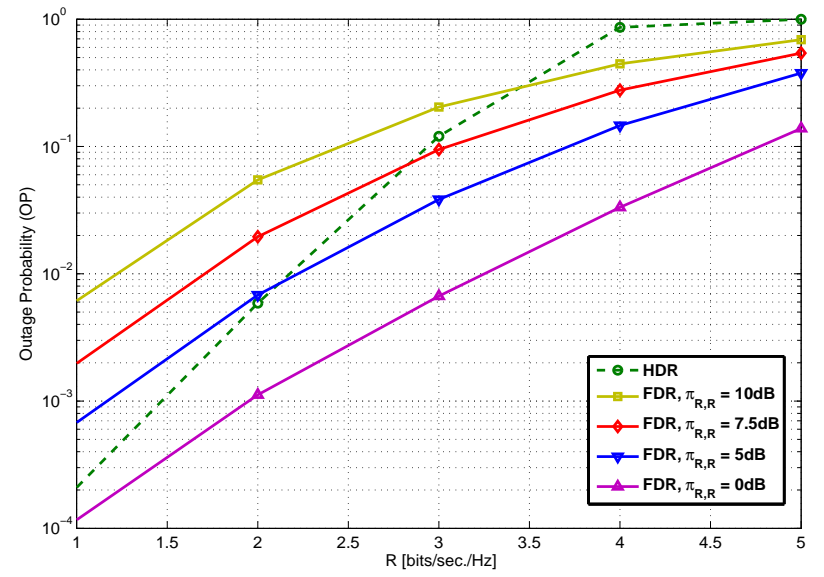

Fig. 5. Outage probability of half-duplex and full-duplex relaying vs. R over asymmetric $\kappa-\mu$ and $\eta-\mu$ fading channels for $\mu_{S, R}=\mu_{R, D}=2, \eta_{R, D}=$ $1, \kappa_{S, R}=2 \mathrm{~dB}$ for different values of $\bar{\gamma}_{R, R}=\pi_{R, R}$ without direct link.

asymmetric HD and FD relay systems under similar fading conditions with $\mu_{S, R}=\mu_{R, D}=2, \eta_{R, D}=1, \kappa_{R, D}=4.5$ $\mathrm{dB}$ for average SNR of $25 \mathrm{~dB}$ and different values of the relay self interference in the case of FD relaying. It is shown that the HD system tends to be in complete outage i.e. $P_{\text {out }}=1$ when $R=4.5 \mathrm{bps} / \mathrm{Hz}$ while the FDR mode performs relatively well for all considered signal strengths of the RSI. In other words, the outage of HDR occurs faster than the FDR system as the spectral efficiency increases. This is because, in order to support the same value of $R$, the instantaneous rate of $\mathrm{HD}$ transmissions must be twice as high as the FD system. It is also noticed that for relatively high values of $R$ e.g. for $3.5 \mathrm{bps} / \mathrm{Hz}$, the FD transmission outperforms its HD counterpart even under strong self-interference of $10 \mathrm{~dB}$. 


\section{CONCLUSiON}

This paper analyzed the outage probability of dual-hop regenerative full-duplex (FD) relay system with relay selfinterference over asymmetric generalized multipath fading channels. Novel analytic expressions were derived for the overall outage probability based on an alternative representation of the cumulative distribution function of the $\eta-\mu$ distribution. It was shown that the performance of the FD system is affected significantly by the fading conditions in each communication path and by the relay self interference. Furthermore, when compared to the corresponding half-duplex relaying scheme, it is shown that the achieved FD OP performance is substantially better, particularly in high target spectral efficiency.

\section{APPENDIX}

\section{PROOF OF EQUATION (10)}

In the considered scenario one obtains

$$
F_{Z}(z)=\operatorname{Pr}(Z \leq z)=\operatorname{Pr}\left(\frac{X}{Y+1} \leq z\right)
$$

According to [26], the CDF of $\mathrm{Z}$ can be expressed as follows:

$$
\begin{aligned}
F_{Z}(z) & =\int_{y=0}^{\infty} \operatorname{Pr}\left(\frac{X}{y+1} \leq z\right) f_{Y}(y) \mathrm{dy} \\
& =\int_{y=0}^{\infty} \operatorname{Pr}(X \leq z(y+1)) f_{Y}(y) \mathrm{dy}
\end{aligned}
$$

which yields

$$
F_{Z}(z)=\int_{y=0}^{\infty} F_{X}(z(y+1)) f_{Y}(y) \mathrm{d} y
$$

where $F_{X}(z(y+1))$ and $f_{Y}(y)$ are the CDF and PDF of the RVs $X$ and $Y$, respectively.

\section{ACKNOWLEDGEMENTS}

This work was supported by the Finnish Funding Agency for Technology and Innovation (Tekes) under the project entitled "Energy-Efficient Wireless Networks and Connectivity of Devices-Systems (EWINE-S)", by the Academy of Finland under the projects No. 251138 "Digitally-Enhanced RF for Cognitive Radio Devices and No. 284694 "Fundamentals of Ultra Dense 5G Networks with Application to Machine Type Communication" and by the National Nature Science Foundation of China Project "Grant No. 61471058".

\section{REFERENCES}

[1] M. Dohler and Y. Li, Cooperative Communications: Hardware, Channel and PHY-1 ${ }^{\text {st }}$ edn. John Wiley \& Sons, 2010.

[2] M. K. Fikadu, P. C. Sofotasios. M. Valkama, Q. Cui, and G. K. Karagiannidis, "Exact Error Analysis and Energy-Efficiency Optimization of Regenerative Relay Systems under Spatial Correlation," IEEE Trans. Vehic. Technol., Accepted for Publication.

[3] Z. Mo, W. Su, S. Batalama,and J. D. Matyjas," Cooperative communication protocol designs based on optimum power and time allocation," IEEE Trans. Wireless Commun., vol. 13, no. 8, 4283-4296, Aug. 2014.

[4] G. C. Alexandropoulos, A. Papadogiannis, and P. C. Sofotasios, "A comparative study of relaying schemes with decode-and-forward over Nakagami- $m$ fading channels," Hindawi J. Comp. Netw. Commun., vol. 2011, Article ID 560528, Dec. 2011.
[5] M. Xia and S.Aissa, "Moments based framework for performance analysis of one-way/two-way CSI-assisted AF relaying," IEEE J. Sel. Areas Commun, vol. 30, no. 8, 1464-1476, Sep. 2012.

[6] M. K. Fikadu, P. C. Sofotasios, M. Valkama, and Q. Cui, "Analytic performance evaluation of $M-\mathrm{QAM}$ based decode-and-forward relay networks over enriched multipath fading channels," in IEEE WiMob '14, Larnaca, Cyprus, Oct. 2014, pp. 194-199.

[7] K. Ho-Van, P. C. Sofotasios, S. Freear, "Underlay Cooperative Cognitive Networks, with Imperfect Nakagami $-m$ Fading Channel Information and Strict Transmit Power Constraint," IEEE KICS Journal of Communications and Networks, vol. 16. no. 1, pp. 10-17, Feb. 2014.

[8] G. C. Alexandropoulos, P. C. Sofotasios, K. Ho-Van, S. Freear, "Symbol error probability of DF relay selection over arbitrary Nakagami- $m$ fading channels," HINDAWI Journal of Engineering, (Invited Paper), Article ID 325045, 2013.

[9] B. P. Day, A. R. Margetts, D. W. Bliss, and P. Schniter, " Full-duplex MIMO relaying: Achievable rates under limited dynamic range," IEEE J. Sel. Areas Commun., vol. 30, no. 8, pp. 1541-1553, Sep. 2012

[10] H. Alves, G. Fraidenraich, R. Souza, M. Bennis, and M. Latva-aho, "Performance analysis of full duplex and selective and incremental half duplex relaying schemes," in Proc. IEEE WCNC, Shanghai, China, Apr. 2012, pp. 771-775.

[11] T. Kwon, S. Lim, S. Choi, and D. Hong, "Optimal duplex mode for DF relay in terms of the outage probability," IEEE Trans. Veh. Technol., vol. 59, no. 7, pp. 3628 - 3634, Sep. 2010.

[12] H. Alves, D.B. da Costa, R. D. Souza, and M. Latva-aho, "Performance of block-Markov full duplex relaying with self interference in Nakagami -m fading," IEEE Wireless Commun. Lett., vol. 2, no. 3, pp. 311-314, June 2013.

[13] M. Khafagy, A. Ismail, M.-S. Alouini, and S. Aissa, "On the outage performance of full-duplex selective decode-and-forward relaying," IEEE Commun. Lett., vol. 17, no. 6, pp. 1180-1183, June 2013.

[14] T. Riihonen, S. Werner, and R. Wichman, "Hybrid full-duplex/halfduplex relaying with transmit power adaptation," IEEE Trans. Wireless Commun., vol. 10, no. 9, pp. 3074-3085, Sep. 2011.

[15] P. C. Sofotasios, and S. Freear, "The $\alpha-\kappa-\mu /$ gamma composite distribution: A generalized non-linear multipath/shadowing fading model," IEEE INDICON '11, Hyderabad, India, Dec. 2011.

[16] P. C. Sofotasios, T. A. Tsiftsis, M. Ghogho, L. R. Wilhelmsson and M. Valkama, "The $\eta-\mu$ /inverse-Gaussian Distribution: A novel physical multipath/shadowing fading model,' in IEEE ICC '13, Budapest, Hungary, June 2013.

[17] P. C. Sofotasios, T. A. Tsiftsis, K. Ho-Van, S. Freear, L. R. Wilhelmsson, and M. Valkama, "The $\kappa-\mu$ /inverse-Gaussian composite statistical distribution in RF and FSO wireless channels," in IEEE VTC '13 - Fall, Las Vegas, USA, Sep. 2013, pp. 1-5.

[18] P. C. Sofotasios, T. A. Tsiftsis, Yu. A. Brychkov, S. Freear, M. Valkama, and G. K. Karagiannidis, "Analytic Expressions and Bounds for Special Functions and Applications in Communication Theory," IEEE Trans. Inf. Theory, vol. 60, no. 12, pp. 7798-7823, Dec. 2014

[19] S. Majhi, Y. Nasser, J. F. Helard, and M. Helard, "Performance analysis of repetition-based decode-and-forward relaying over asymmetric fading channels," in Proc. IEEE PIMRC, Turkey, Sep. 2010, pp. 362-367.

[20] H. A. Suraweera, G.K. Karagiannidis, and P. J. Smith,'Performance analysis of the dual-hop asymmetric fading channel," IEEE Trans. Wireless Commun., vol. 8, no. 6, pp. 2783-2788, June 2009.

[21] K. P. Peppas, G. C. Alexandropoulos, P. T. Mathiopoulos, "Performance analysis of dual-hop AF relaying systems over mixed $\eta-\mu$ fading channels," IEEE Trans. Veh. Technol, vol. 62, no. 7, pp. 3149-3163, Sep. 2013.

[22] M. D. Yacoub, "The $\kappa-\mu$ distribution and the $\eta-\mu$ distribution," IEEE Ant. Propag. Mag., vol. 49, no. 1, pp. 68-81, Feb. 2007.

[23] I. S. Gradshteyn, and I. M. Ryzhik, Tables of Integrals, Series, and Products $-7^{\text {th }}$ edn. Academic Press, 2007.

[24] D. B. da Costa, and M. D. Yacoub, "Accurate closed-form approximations to the sum of generalized random variables and applications," in Proc. IEEE WCNC, Las Vegas, NV, USA, 2008, pp. 785-790.

[25] H. Alves, G. Fraidenraich, R.D. Souza, M. Bennis, and M. Latva-aho, "Performance analysis of full duplex and selective and incremental half duplex relaying schemes," in Proc. IEEE International Conference on wireless Commun. and Ntw.: PHY and Fundamentals, 2012, pp. 771-775.

[26] A. Papoulis, S.U. Pillai, Probability, Random Variables and Stochastic Processes, $-4^{\text {th }}$ edn. McGraw-Hill, 2002.

[27] A. H. Nuttall, "Some Integrals Involving the $Q_{M}$ Function," IEEE Inf Theory, vol. 21, no. 1, pp. 95-96, 1975. 